\title{
Full Geo-localized Mobile Video in Android Mobile Telephones
}

\author{
Elsa Macías ${ }^{1}$, Hanna Abdelfatah ${ }^{2}$, Alvaro Suárez ${ }^{1}$ and Alejandro Cánovas ${ }^{3}$ \\ ${ }^{1}$ Concurrency and Architecture Group (GAC) \\ Department of Telematic Engineering \\ University of Las Palmas de Gran Canaria, Spain \\ \{emacias, asuarez\}@dit.ulpgc.es \\ ${ }^{2}$ Graduated Student at University of Las Palmas de G.C. \\ hanna.bint101@estudiantes.ulpgc.es \\ ${ }^{3}$ Instituto de Investigación para la Gestión Integrada de Zonas Costeras (IGIC) \\ Universidad Politécnica de Valencia, Spain \\ alcasol@posgrado.upv.es
}

Received: April 10, 2011 Accepted: April 30, $2011 \quad$ DOI: 10.5296/npa.v3i1.641

\begin{abstract}
The evolution of mobile telephones have produced smart devices that not only allows the mobile user to talk but also to use a lot of telematic services. High quality photos and videos are produced by smart mobile telephones. The Global Positioning System available in the Mobile telephones allows the user to tag their photos and videos. There are several photo and integral video tagging mobile software but there is not a mobile application that allows the mobile users to tag the full video frames. This full tagging process allows the mobile user to tag independent video frames in order to explode the photo-video properties of the integral video. In this paper we present a mobile application and a Server application that allow the mobile user to full tag the mobile videos and share them with other users (registered in the Server). We present some tradeoffs present in the design of the tagging process.
\end{abstract}

Keywords: Mobile telephones, WiFi, Android, Mobile Video, Geolocalization, GPS. 


\section{Introduction}

Mobile telephones have experienced a great evolution in the recent past years. They are provided different wireless communication interface cards. One of these interface cards is the Wireless Fidelity (WiFi) [1] and another is the Global Positioning System (GPS) [2]. In the past 20 years it has been a great acceleration in the development of geo-location technologies. They originally were deployed to meet needs in the military field. Thanks to research conducted, numerous satellites were launched into Earth's atmosphere, in order to locate the position of an object on the surface of the Earth, using the triangulation principle [3].With regard to localization projects carried out, without a doubt the most important is the GPS. Initially, the civil enforcement GPS devices were designed specifically to find the location of the system that incorporates them or to have and use stored information. Over the years, and the incredible development of mobile telephones, culminating in the third and fourth generation of these devices, it was entering the reception and processing of GPS signals to these terminals.

Mobile telephones also include powerful video cameras [4]. These video cameras allow the mobile telephones to register long time videos. These videos can be stored in the mobile telephone memory or can be streamed to a Video Server (normally allocated in Internet). Geo-localization in mobile telephones is a common technology that allows mobile users locate their friends (normally under a Social Network). Massively used services like Google Maps normally use the geo-localization to allow a mobile user to move in an unknown place. A step forward in geo-localization services is to allow the mobile user to insert a photo in typical maps services in order to show the exact position in which that photo was taken (geo-tagged photo). Among others, services like Google Earth allow the specification of a route followed by a user. Attached to that route the user can tag photos and videos. The application of this is explained (but not implemented) in [5]. One service that has not had much impact has been relative to the location of full video geo-tagging recorded from a mobile telephone. This is undoubtedly due to the difficulty involved geo-tagging each video frame (corresponding to images), using the reading of the location of capture device and synchronize this information (obtained from GPS receiver incorporated) with the recording frequency. You should make use of orientation sensors that incorporate most of these devices, to use mathematical tools to define the coordinates of each image. However, the appeal of geo-tagged full video sharing and the possibility to search by criteria (streets, cities, etc.) opens the door to many applications. An example of this is CityTube [6]. However, this Google application does not use exactly the geo-location of the frames; it uses other criteria. The aim of this work is to design and implement a mobile application that tags full video frames using the localization sensor of the mobile telephones. In contrast with other works we generate a full tag video which opens a wide range of new applications.

The structure of this paper is the following: In section 2 we will present the related work taking into account the localization problem and the tagging problem. In Section 3 we will present the tradeoffs of design of the tagging system. In Section 4 we will present briefly the communication protocol design issues. In Section 5 we will present the description of the mobile application. In Section 6 we will present some conclusions and future work. 


\section{Related Work}

Nowadays, mobile devices not only use integrated receivers that pick up signals from satellite navigation systems but also make use of other techniques that use less resources and offer greater coverage. These alternative techniques are called local positioning systems. Among the existing, the most important are Assisted GPS (A-GPS) [7], WiFi Positioning Systems(WPS) [8] and Hybrid Positioning Systems(XPS) [9].

A-GPS is a system that decreases the start time of techniques based on satellite tracking as GPS. This system arose from the need, which was at the time, to track calls made to emergency service for the address of the person or persons in distress if it had not been possible to provide it. Therefore, its use is intended for mobile phones. The conventional GPS only uses radio signals from satellites. A-GPS also uses cellular networks to locate. This allows more efficiency to determine locations by accessing the satellite data more quickly and better in weak signal conditions. There are two types of A-GPSs: those who work on-line and off-line. The first, from its cellular network information, facilitating the server, obtain the necessary data from satellites to calculate positions, plus additional data such as orbital parameters and ionospheric conditions. They also allow updated information all the time and need to communicate with the server every time a geo-position is referenced. The latter, however, collect information from the server periodically and store assistant in the terminal. Each time the user is going to make a positioning it uses this stored information. The main disadvantage of this method is that the stored data may be outdated but is faster than the previous.

A-GPS is a system that works pretty well even in indoor environments. The cellular network coverage is less than the WiFi network, indoors. For this reason WiFi Positioning System (WiFi PS) raised in order to solve the positioning in situations of very low coverage, as indicated above, taking advantage of the remarkable increase in access points in urban environments in recent years. The company Skyhook Wireless [10] is a leader in the market for this type of service, maintaining a public database (with information about identifiers of access points with corresponding geographic location) that can be accessed using an Application Programming Interface (API) and providing an information server nearest access point, which can be determined from a telephone with WiFi wireless network interface card.

Hybrid positioning systems use multiple technologies to determine location coordinates of a mobile device. This technology combines GPS' signal (which are normally the largest contributor in the process), mobile $3 G$ stations' signal, wireless Internet's signal, sensors, Bluetooth and other local positioning systems.

In this paper we only use the localization provided by GPS traditional system. We are interested in the geo-localization of video frames with latitude and longitude tags provided by the mobile telephone's GPS. The importance of our work is guaranteed by the reflections shown in [11]: a new spectrum of applications is opened mixing the geo-tagging services with the existing for photo and videos (Flickr, youtube, Twitter...). 
An example of photo (taken with a mobile telephone) and geo-tagging services is presented in [12]: the user just snaps a photo which is sent to an application server over a Universal Mobile Telecommunication System (UMTS) network and then forwarded to a Server. That Server calculates the position of the user and stores it using an eXtensible Markup Language (XML) format archive. Then, the Server sends back the position to the mobile telephone which visualizes the position of the user in a map. Our mobile application calculates the position of the user (the mobile telephone) in the mobile telephone. This is possible in actual smart mobile telephones. With this we save wireless bandwidth, Server resources (memory and processing) and time (we skip the mobile telephone-Server communication loop).

Another photo tagging application is shown in [13]. Photos are tagged with tags that indicate the GPS location and time they are taken (these tags are used to derived other context-based metadata such as weather conditions and daylight conditions). The photos and the tags are stored in the mobile telephone memory in order the mobile user to seek them easily. This can be done for a restricted number of photos due to memory restrictions. This technique is not appropriated for full video tagging due to the amount of video frames could be very big. For this reason we prefer to publish the tagged full video frames in a Server. Moreover, this is appropriated to easily share the videos among users.

There are several initiatives working with the tagging of video using geolocation information for such tagging process. In fact, there are different ways to geo tag the video content. On platforms where users upload videos usually it is done by associating geolocation video content to the user's geographic location (using IP)[14] and verifying the contents of the video (Google Earth, GoogleMaps and CytiTube). On one hand, on these platforms the location of multimedia content can be performed requesting to the user to upload the video and the global tags (those that qualify the entire video). On the other hand, there is the geolocation of multimedia content from the geographical coordinates of the camera that was used to record a particular video [15]. Later, you can play the video and capture on a map the route crossed during the recording (Rally Maps [16], VidMap [17]). This technique is also being used for videos that are transmitted by live streaming and let live geolocation (Qik [18], Ipoki [19]).

Actually it is a hot topic the utilization of GPS in virtual reality and augmented reality to model 3D environments. In [20], it is presented a brief of some works related to this research area. In concrete, they center the overview in the visualization of personal videos in 3D virtual environments and $3 \mathrm{D}$ video re-construction. We are not centered in $3 \mathrm{D}$ only. Once the video is uploaded to the Server, one post-processing could be to do a $3 \mathrm{D}$ virtual environment visualization but other processes can also be done.

In this paper we have attempted to improve the geo-location technique associating each video frame specific geographic coordinates. To this end, besides the geographical coordinates of the mobile telephone's video camera has had to make use of other values (angular values read form the telephone sensors) to calculate the geographical coordinates of the objects aimed at a certain distance from the camera. This is not an easy work due to some 


\section{Macrothink}

tradeoffs will be present at the time to do the tagging process of each individual video frame. We have identified some of them and tried to solve the problem efficiently. But in general this problem needs more work in order to save time and memory space. We also have tried to save the needed wireless bandwidth in the communication between the mobile telephone and the Server.

\section{Tradeoffs in Mobile Video Geo-localization}

In this section we review the details of the Android platform respecting to the: Definition of the coordinates systems (latitude and longitude) and the reading of the values provided by the GPS.

\subsection{Android Coordinates System}

Android uses a system of three dimensions Cartesian coordinate that is allocated in the geometric center of the mobile telephone. We name these coordinates Vertical ( $Y$ ), Horizontal $(X)$ and Depth $(Z)$. But these coordinates change with respect to the Earth's surface depending on the relative position of the mobile telephone respecting to the Earth Surface. In Figure 1, this coordinates system is shown. These coordinates are defined to calculate in each time the orientation of the device as a function of angular values. These angular values are named pitch, roll and azimuth. Android uses the pitch, roll and azimuth data for the tilt and orientation of the device and the cardinal direction respectively. These three angular measurements are derived from the orientation sensors and digital compass which have most of the mobile telephones that can run Android platform.

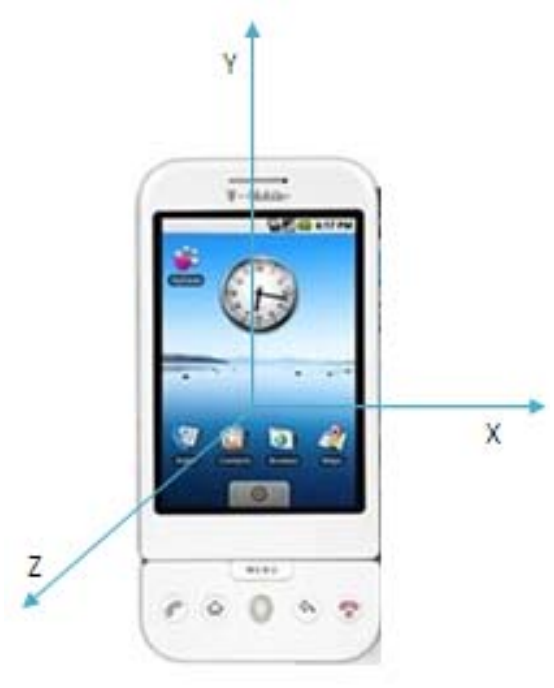

Figure 1. Android Coordinate System.

The azimuth is defined as the angle between the direction of true North and the Y axis projection of the mobile telephone on the floor (Earth surface), because the geographical North is defined parallel to the ground. It can also be defined as the angle between true North 


\section{Macrothink}

and the cardinal direction pointed to the mobile telephone's camera. It takes values between $0^{\circ}$ and $359^{\circ}$. These values agree with those who take the azimuth marine, where $0^{\circ}$ indicate the north, $90^{\circ}$ the east, $180^{\circ}$ the south and $270^{\circ}$ the West. Therefore, from this values can be obtained cardinal direction the device camera is pointing at any time. The pitch is defined as the angle of rotation around the $\mathrm{X}$ axis of the mobile telephone, with positive values when the top of the phone looks to the ground. It takes values in the interval $\left[-180^{\circ}\right.$ and $\left.180^{\circ}\right]$. The roll is defined, in turn, as the angle of rotation around the $\mathrm{Y}$ axis of the mobile telephone, with positive values when the rotation is toward the $\mathrm{X}$ axis of the mobile telephone (values between $-90^{\circ}$ and $90^{\circ}$ ). Importantly, both the pitch and the roll are calculated from the default position of the mobile telephone (on the floor with the $\mathrm{Z}$ axis parallel to the axis of elevation of the Earth).

\subsection{Reading the Values Provided by the GPS in the Android Mobile Telephone}

The main novelty of our mobile application is that it tags the full mobile video. That is, our mobile application tags each video frame with the values provided by the GPS system of the Android platform. This is not a simple task. In Fig. 2 we show a schematic graphic of the tagging process. When the video recording system starts, the GPS must previously be set up and contacted with the satellites needed to make the localization. This time is not taken into account due to it is a requirement to start the video registering. But once started the tagging process each video frame must be tagged with the values provided by the GPS system. Each video frame will be assigned with its $X, Y$ and $Z$ values as indicated in Fig. 2.

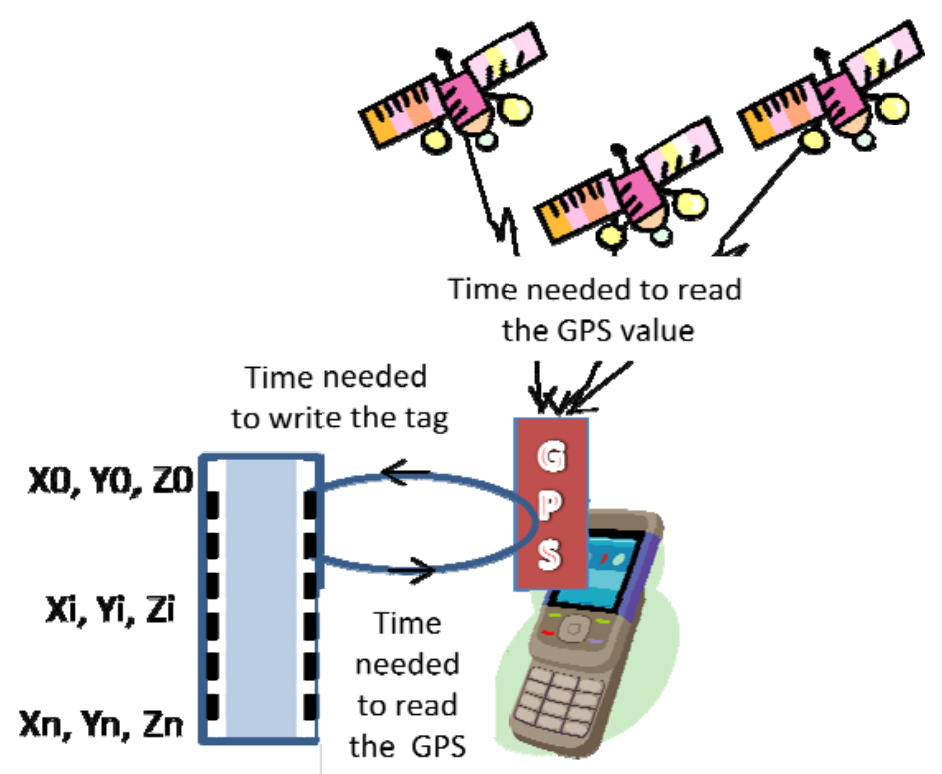

Figure 2. Graphical view of the time needed to tag the video frames. 


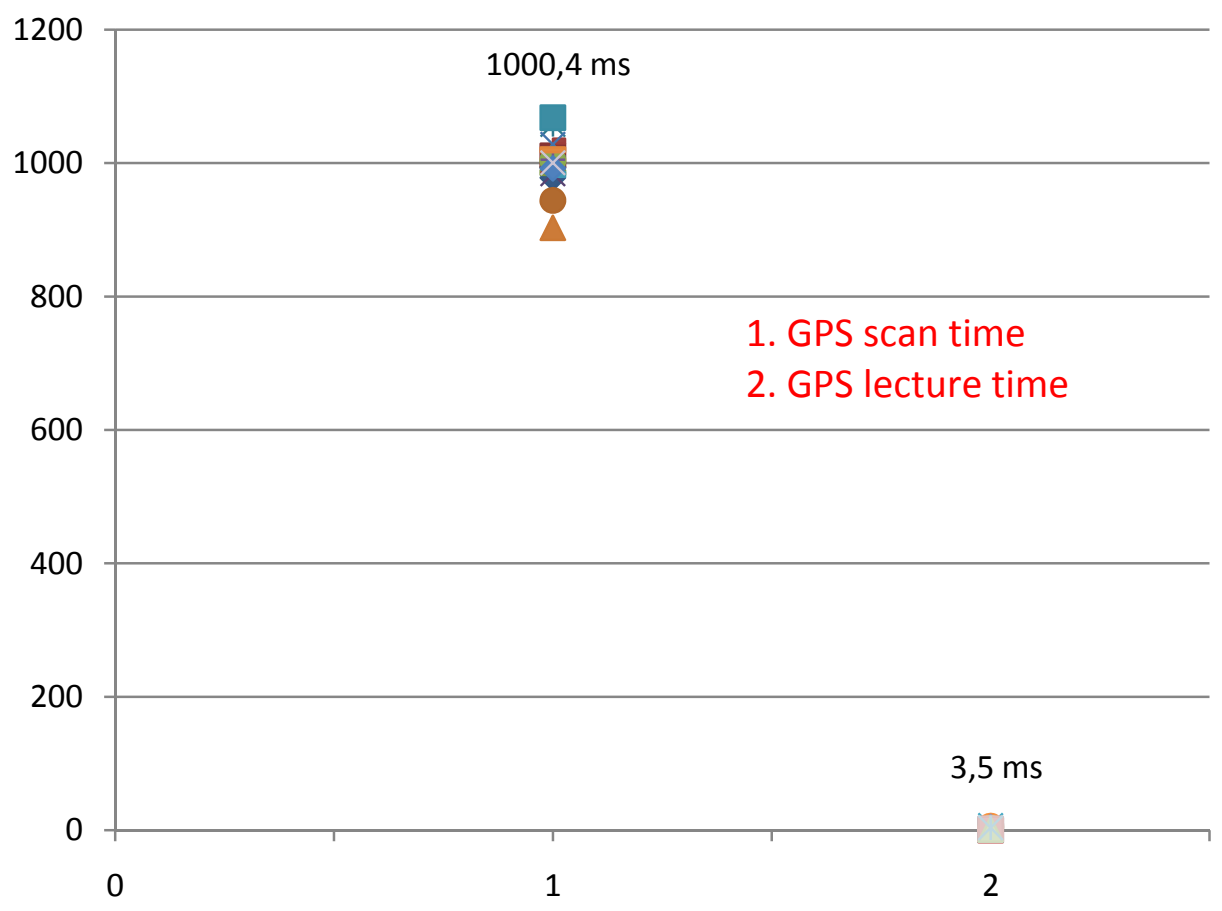

Figure 3. Different measurements of the time needed to read the GPS values.

We need to tune the following parameters:

- The amount of frames recorded by the mobile video camera. A typical video mobile camera is able to capture 25 frames per second (fps). But there are mobile video cameras that are able to read up to $30 \mathrm{fps}$.

- The time needed to read a particular value of the GPS from the GPS and to read it in the mobile telephone. While the first value is around $1 \mathrm{~s}$, the second value is around $10 \mathrm{~ms}$ as indicated in Fig. 3 for 20 different measurements.

- The time needed to tag a particular video frame. This is the time needed to register in the Secure Digital memory card the tags. This time is very small compared with the time needed to read a particular GPS value. And can be despised.

As a result of the above analysis, there is an inconvenient with the video frames tagging software in the mobile telephone: not all the video frames will be annotated with the corresponding GPS tag. That is, it is very hard to annotate individual video frames with the exact value of longitude and latitude in which it was captured by the mobile video camera. In practice, another important aspect is that modern Coder-Decoders (CODECs) do not generate fixed length video frames. For this reason, it is hard to dimension the amount of time needed to tag a particular video frame. For example Moving Picture Experts Group 4 (MPEG4) 
CODECs normally generate video frames that are a real picture and subsequent differences video frames that contain only differences with respect to the anterior frames. For these reasons, we propose to tag different video frames with the same GPS' value. The limitation of this is when the mobile telephone is moving with high speed. In such case, there can be a considerable error of tagging process.

In Fig. 4 we show the overall procedure to tag the video frames.

A graphical specification of the geo-tagging is shown in Fig. 5. It is shown that for a determined trajectory, every video frame the coordinates file stored the tags.

\section{Brief Description of the Mobile Telephone and Server Communication Protocol}

Once the mobile telephone has stored the full video frames tagged with GPS values it achieves the communication protocol described in Fig. 6. In that Fig. we have named the mobile telephone as the Client (the protocol is a typical Client-Server protocol). Basically, the client will send a registering request to the Server (Query registration message). The Server will send a Registration start message to the Client. After this, the Client will send a typical authentication message (User + Pass). After this, if the Server accepts that registration request (according to the information stored in its data base), then it will send an accept message to the Client. On the contrary it will send a reject message to the Client. This protocol uses the encryption and de-encryption process described in [21]. The Client and the Server use private and public keys. From the public key of the receiver the sender will cipher the message. Once the message has been sent it will be only de-ciphered using the private key. 


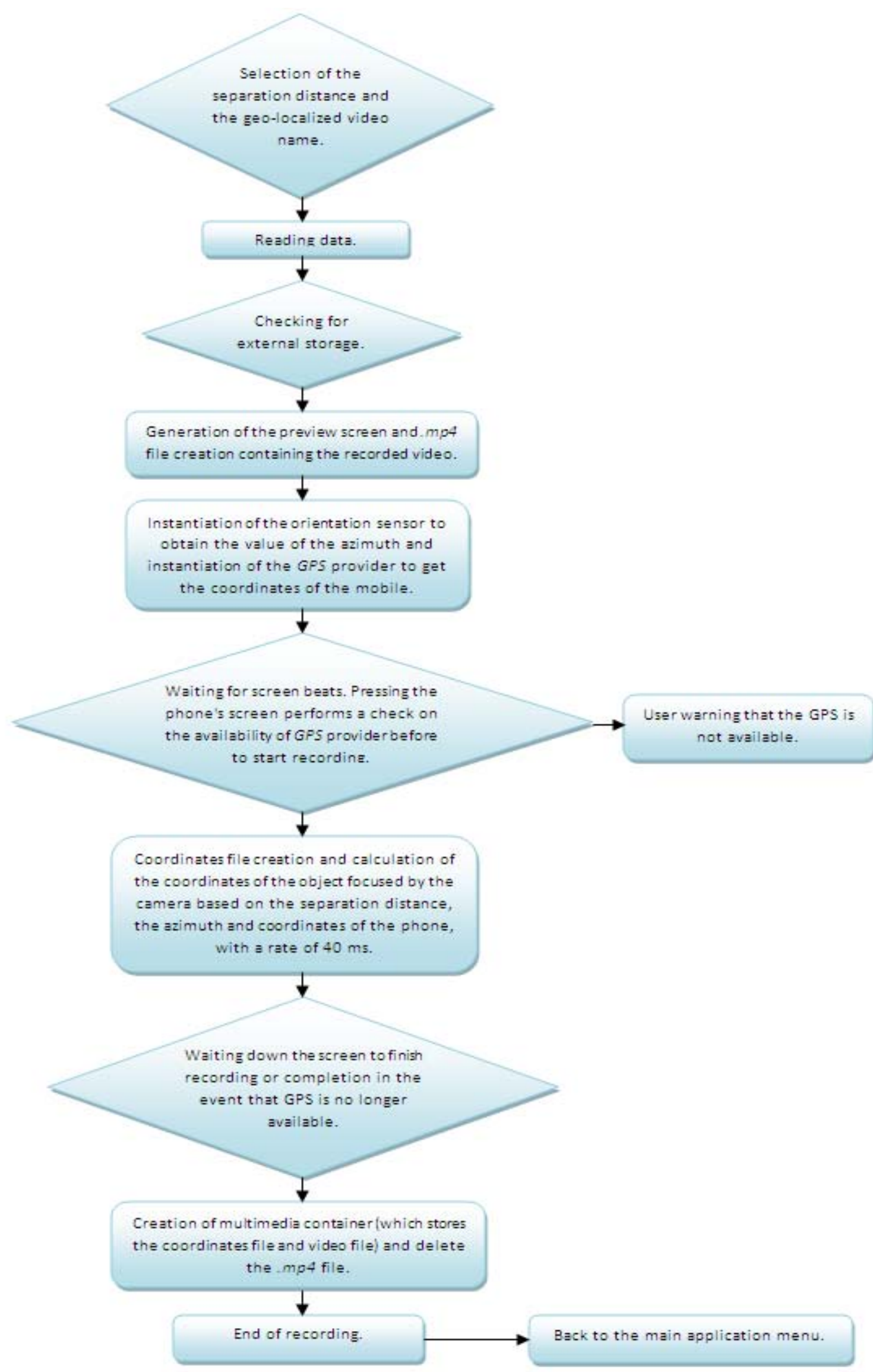

Figure 4. Chart of procedure to geo-tag videos. 
Android terminal recording sequence. GPS information is captured with the provider used and the video frames with the camera device. The recording is stopped if the GPS is not available.
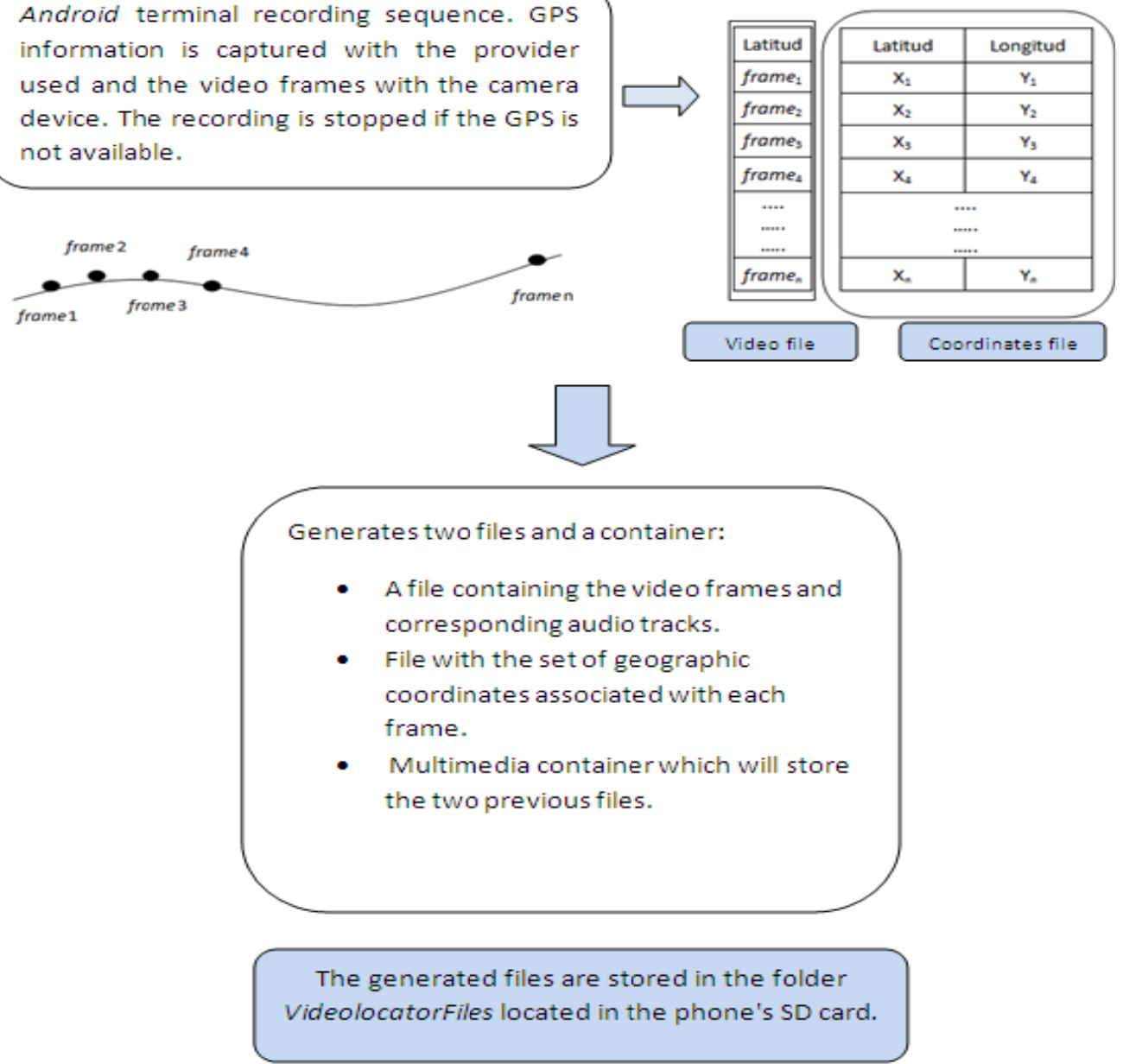

Figure 5. Graphical specification of the geo-tagging of videos frames.

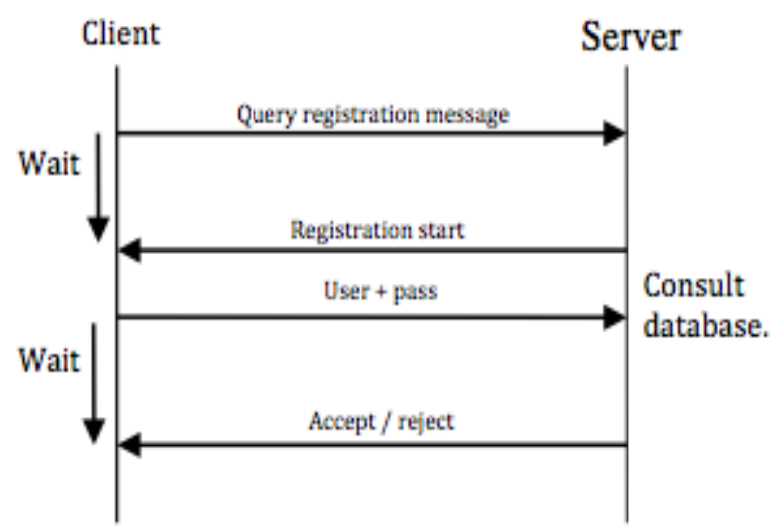

Fig 6. Client registration protocol.

In the Fig. 7 is shown the format of the Query registration message which contains: The 
client transport protocol Port and the Client Internet Protocol (IP) address. The Port and IP address of the Server, the Client's Public Key and the kind of message (Query registration message).

\begin{tabular}{|c|c|c|c|c|c|}
\hline $\begin{array}{c}\text { Client } \\
\text { IP }\end{array}$ & $\begin{array}{c}\text { Client } \\
\text { Port }\end{array}$ & $\begin{array}{c}\text { Server } \\
\text { IP }\end{array}$ & $\begin{array}{c}\text { Server } \\
\text { Port }\end{array}$ & $\begin{array}{c}\text { Client } \\
\text { public key }\end{array}$ & $\begin{array}{c}\text { Query registration } \\
\text { message }\end{array}$ \\
\hline
\end{tabular}

Fig 7. Client query registration message.

In the Fig. 8 is shown the format of the Registration Start message. This message is sent encrypted using the Client's public key. It contains: the Ports of the Client and the Server, the IP address of the Client and the Server and Server's Public Key. The Client will de-encrypt this message using its private key and it will send the new message containing the username and the password.

\begin{tabular}{|c|c|c|c|c|c|}
\hline $\begin{array}{c}\text { Server } \\
\text { IP }\end{array}$ & $\begin{array}{c}\text { Server } \\
\text { Port }\end{array}$ & $\begin{array}{c}\text { Client } \\
\text { IP }\end{array}$ & $\begin{array}{c}\text { Client } \\
\text { Port }\end{array}$ & $\begin{array}{c}\text { Server public } \\
\text { key }\end{array}$ & $\begin{array}{c}\text { Registration } \\
\text { start message }\end{array}$ \\
\hline
\end{tabular}

Fig 8. Server registration start message.

In Fig. 9, the full tagged video frames communication (once the Client is well registered in the Server) is shown:

- Firstly, the Client will send an open connection message. It uses a blocked Transport Control Protocol (TCP) socket. So, it will wait the Server confirmation message.

- Secondly, the Server will acknowledge the connection. The Server will confirm the open connection message just in case the corresponding TCP port to be opened.

- Thirdly, the Client will stream the full tagged video frames (that is, the video and the archive with tags).

- Fourthly, the Server will send a finish message to the client when the video communication will be finished.

- Finally, the Client will acknowledge the reception of the above message. In this moment the connection will be terminated.

As a result, it can be detected that the video is not sent in encrypted mode to the Server: the only messages the Client and the Server encrypt or de-encrypt are the messages corresponding to the initial authentication of the Client in the Server. 


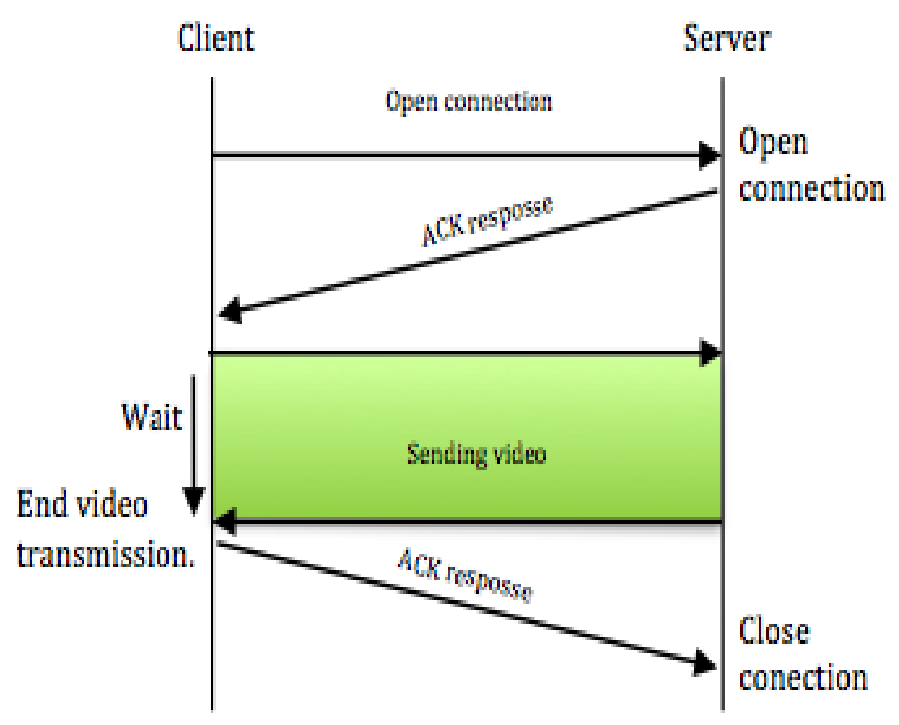

Fig 9. Video transmission protocol.

\section{Description of the Geo-localized Mobile Video Application}

The developed Application is shown in Fig. 10. It consists of a mobile client application developed in Android, a multi-platform Java Server which connects the mobile application to a database and a file-sharing module that is associated to each user. 


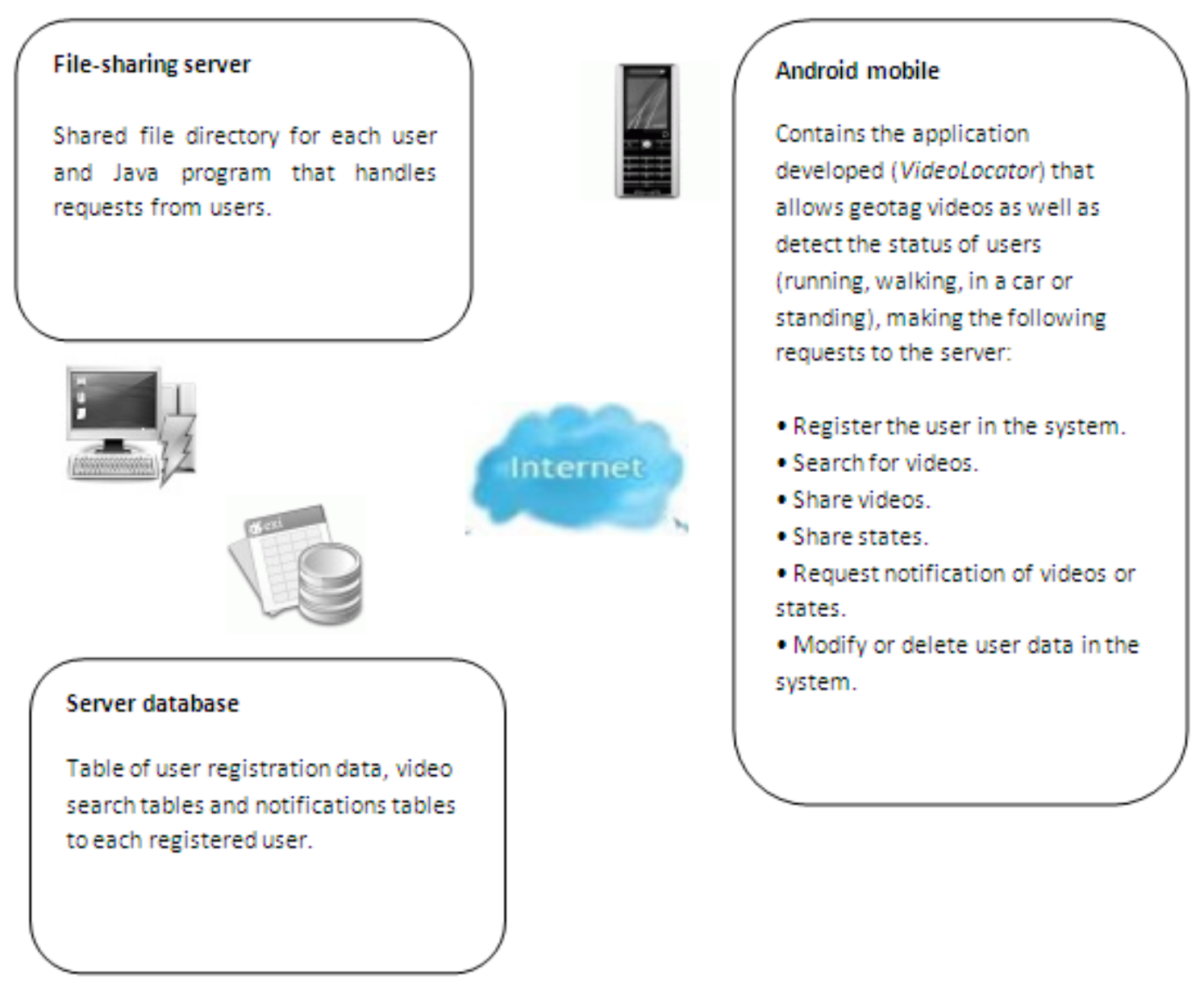

Figure 10. Details of the Mobile Application.

There are some requirements to be accomplished by the mobile application and the Server:

- The mobile client application provides a user interface simple and intuitive, so that any user, without needing to know implementation details of it, can become familiar with its use quickly. In Fig. 11 we show two aspects of the user interface of the mobile application. Fig. 11.a shows a user interface to generate a geo-located video and Fig. 11.b shows the confirmation to the user from the application that the file sent to the server has been properly shared. 


\section{Macrothink}

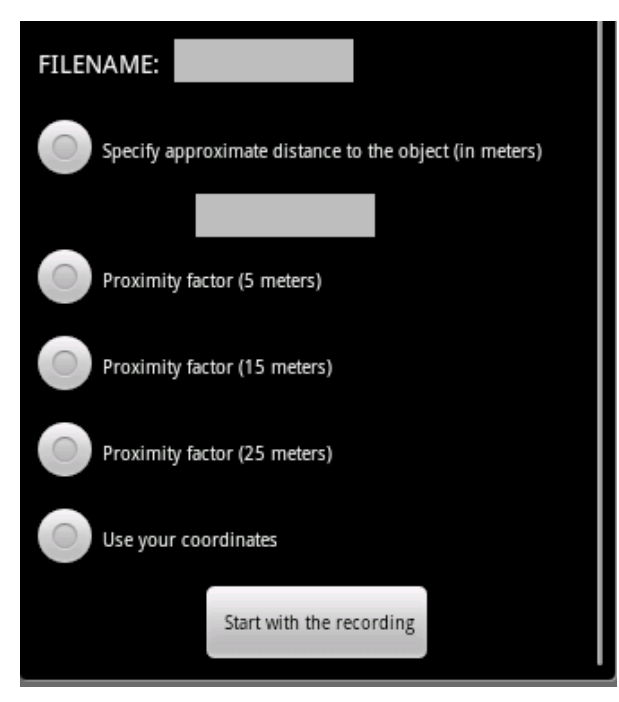

(a)

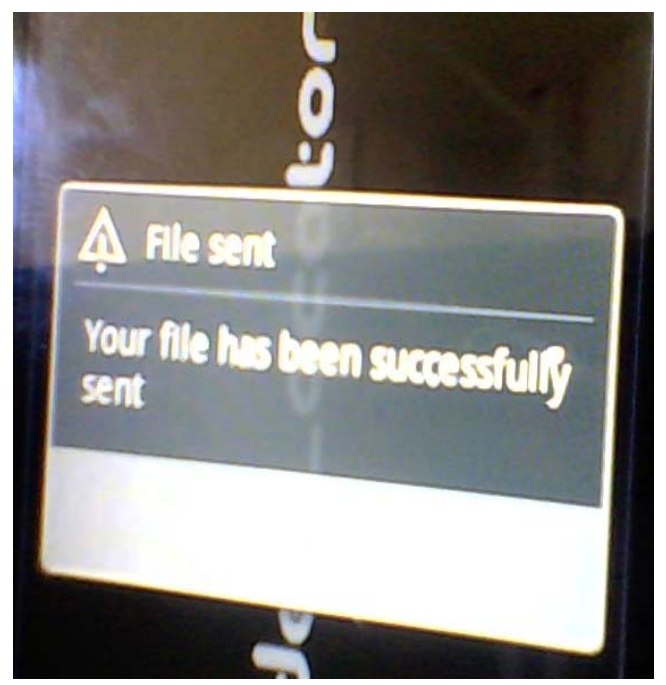

(b)

Figure 11. Two different aspects of the user interface: a) video tagging, b) file sending.

- The application is highly reliable and efficient, ensuring users the assurance that the requested operations have been successful and fastest way possible. It is also compatible with different versions of Android, from 1.6 to 2.2. In addition, it performs multiple operations that require the connection to the Server. In order to do this, the Server needs to keep the user informed of any developments in those connections.

- In addition, the mobile client application (at the beginning of every connection to the Server), first detects whether it is available, informing the user if it will be not available. Also, it shows the user the available WiFi networks, indicating the highest level signal WiFi, suggesting that connects to it, if it was connected to another one. On the other hand, any user of the mobile client application can detect its current state using the tool provided for that purpose. Status detection will be based at the speed that the mobile device has. This speed can be calculated with the corresponding geo-location information. Thus, observing changes in geographical coordinates over time, it can be determined the mobile telephone velocity.

- The server allows the storage of video files and performing searches by criteria in addition to notifying the user of certain events if the user will request it. Similarly, the server, which is written in Java is multi-platform and can be installed in different operating systems (Windows, Linux or Mac OS). It also simulates the behavior of a single social network Server, allowing users to share files and states and notifying them of certain events if they will wish it. To do this, it has a registration system for users in a database, and assigns a memory space for each of them, where they can host their videos. 
Table 1. Codes of requests from the client application to the server.

\begin{tabular}{|c|c|}
\hline Code & Operation \\
\hline "Main" & Verify user data \\
\hline "Register" & User registration \\
\hline "DeleteData" & Delete user from the system \\
\hline "DeleteVidNot" & Delete video notifications \\
\hline "DeleteVidSta" & Delete state notifications \\
\hline "Modify" & Change password \\
\hline "NotificationVidCoor" & Video notification request using coordinates \\
\hline "NotificationVidFile" & Video notification request using coordinates file \\
\hline "NotificationSta" & State notification request \\
\hline "ReceiveVidNot" & Request for receiving registered video notifications \\
\hline "ReceiveStaNot" & Request for receiving status updates \\
\hline "SendFile" & Request for sending multimedia file for sharing \\
\hline "SearchFile" & Request for search video files using coordinates file \\
\hline "SearchCoor" & Video search request by coordinates from Google Maps or user specified \\
\hline "SearchPlacesStreets" & Video search request by a range of coordinates from one place or street \\
\hline "ReceiveSearch" & Request for receiving video search results \\
\hline "ReceiveNotVid" & Request for receiving video notifications results \\
\hline "ReceiveDataSearch" & Request for receiving data information from a particular user whose video is the result of a search \\
\hline "ReceiveDataVidNot" & Request for receiving data information from a particular user whose video is a notification \\
\hline "ReceiveDataStaNot" & Request for receiving data information from a particular user whose state is a notification \\
\hline "UpdateSta" & $\overline{\text { Request status update }}$ \\
\hline "DeleteVidNot" & Delete request of video notifications \\
\hline "DeleteStaNot" & Delete request of status notifications \\
\hline "ListAll" & Request to watch all the videos stored on the server \\
\hline 0 & Code to verify if the server is operating \\
\hline
\end{tabular}

- The mobile client application communicates with the server using Java sockets. To do so through a TCP connection requests to the server using a particular transaction. To this end it uses a series of codes depending on the required operation. Table 1 explains these codes in detail.

Moreover, for each open connection the server must control the flow of data given in the same until the transaction finish. Figure 12 shows the data flow that is generated on the Server when a user requests from his mobile telephone to send a geo-located video. The user, on the other hand requires the application interface to request the desired operation. 


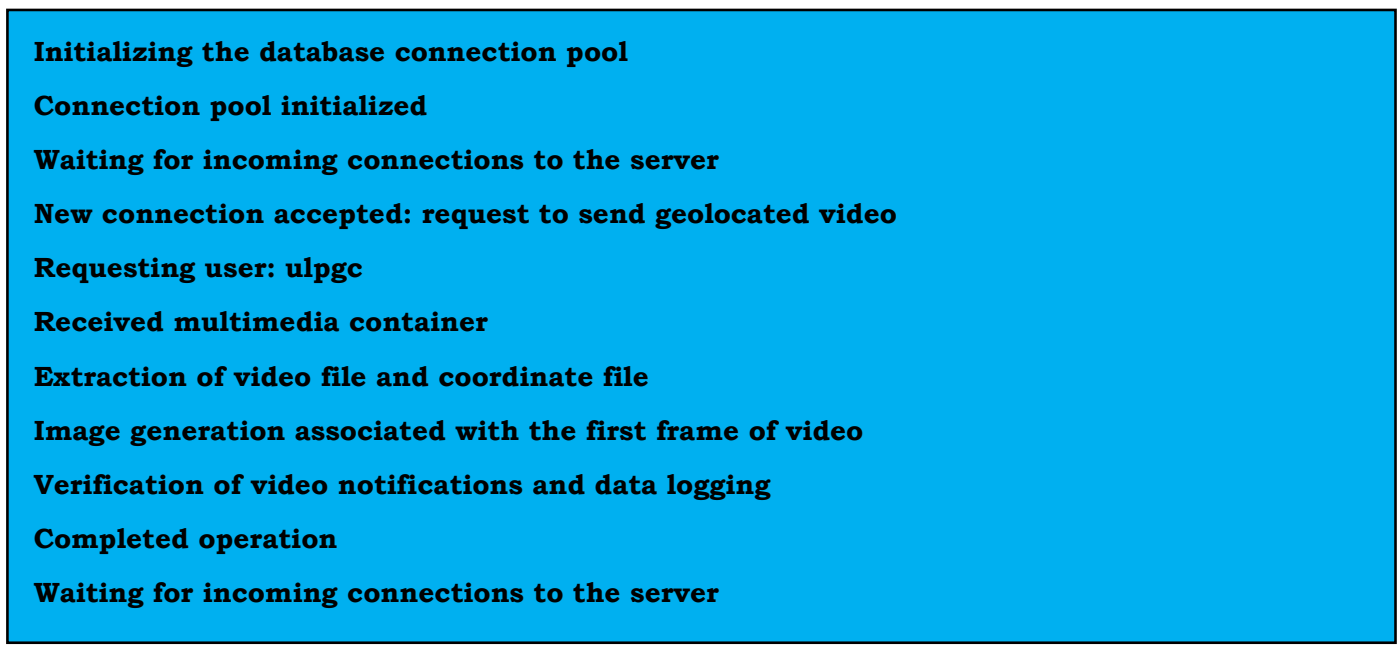

Figure 12. Data flow in the server when a user request and send a geolocated video.

\section{Conclusion and Future Work}

The application of the GPS system to tag the mobile photos and videos is very interesting in our days and open new business opportunities. This is recognized in some recent papers and mobile applications.

There are several application dedicated to time and GPS tagging of photos and entire videos produced by mobile telephones. There are also some new businesses that integrate services like Google Earth or Google Maps and Youtube with video and photo's tagged with GPS. All the above applications and services only consider the entire video tagging. The user must tag manually the entire video before to upload it to the video Server.

In this paper, we have gone one step forward and considered the full video frames tagging process in the mobile smart telephone. This process will produce a better geo-tagging of the video and produce new kind of queries to semantically discover properties of the full video automatically. But this process includes some important tradeoffs that we have reviewed in this paper: it is not easy to tag independent video frames due to the delay associated to the reading of the GPS values in the mobile telephone. Moreover, it is important to consider the real time response of the software and the storing of the tagged video frames in the mobile telephone.

We have implemented our mobile application in an HTC mobile telephoned provided with an android platform. The response of the mobile application is high and the efficiency of the communication protocol is also very high (we save time and wireless communication bandwidth). We have used WiFi technology to measure the performance of the communication system. In the next months the new 4G communication will reach the actual speed of transmission of WiFi technology. So we think our results are very interesting. This is exactly the future work we want to achieve in the next months: To probe with 4G technology. 


\section{References}

[1] Lansford J., Stephens A., Nevo R., "Wi-Fi (802.11b) and Bluetooth: enabling coexistence," Network, IEEE, vol.15, no.5, pp.20-27, Sep/Oct 2001 doi: $10.1109 / 65.953230$

[2] K. Borre, D.M. Akos, N. Bertelsen, P. Rinder, and S. Holdt Jensen, “A Software-Defined GPS and Galileo Receiver”, in Computer Science, Springer e-books. Ed. Birkhäuser Boston: Boston, MA, 2007.

[3] Guillaume M., Yann M., Jo ao F., Hans-Peter S. and István Z., “Enhanced triangulation method for positioning of moving devices”. Unpublished.

[4] Kawamura S., "Capturing images with digital still cameras," Micro, IEEE , vol.18, no.6, pp.14-19, Nov/Dec 1998 doi: 10.1109/40.743680

[5] Application Development for Geo-Referenced Audio Video Recording of the Traffic System Using Mobile Phones.Available at:

http://vladimir.remenar.net/application-development-for-geo-referenced-audio-video-recordin g-of-the-traffic-system-using-mobile-phones/ (last accessed May 3, 2011)

[6] Cytitube. Available at: http://cytitube.com/ (last accessed May 3, 2011)

[7] Frank van D., “A-GPS: assisted GPS, GNSS, and SBAS”, in GNNS technology and applications series, Ed. Artech House: Boston; London, 2009.

[8] Erin-Ee-Lin Lau; Wan-Young Chung; , "Enhanced RSSI-Based Real-Time User Location Tracking System for Indoor and Outdoor Environments," Convergence Information Technology, 2007., pp.1213-1218, 21-23 Nov. 2007 doi: 10.1109/ICCIT.2007.253

[9] Soliman S., Agashe P., Fernanadez I., Vayanos A., Gaal P.andOljaca M., "gpsOne ${ }^{\mathrm{TM}}$ : a hybrid position location system", Sixth Symposium on Spread Spectrum Techniques and Applications (SSSTA, 2000)”. September 2000.

[10] Skyhook Wireless. Available at: http://www.skyhookwireless.com/howitworks/ (last accessed May 3, 2011)

[11] Friedland, G. and Sommer, R., "Cybercasing the joint: On the privacy implications of geo-tagging”. In HotSec, 2010.

[12] Dušan O.andAleš L., "Hyperlinking Reality via Camera Phones”, In Proceedings of the 27th international conference extended abstracts on Human factors in computing systems (CHI EA '09). ACM, New York, USA, 3515-3516. http://doi.acm.org/10.1145/1520340.1520519

[13] Gurrin, C., Jones, G., Lee, H., O'Hare, N., Smeaton, A.F., and Murphy, N., “Mobile 
access to personal digital photograph archives”. In Proceedings of the 7th international conference on Human computer interaction with mobile devices I\& services (MobileHCI '05). ACM, New York, NY, USA, 311-314. doi:10.1145/1085777.1085842 http://doi.acm.org/10.1145/1085777.1085842

[14] Jang-Ping, S. Yu-Cheng, L. Shin-Chih T. , "Location-based IP assignment protocol for ad hoc networks," Sensor Networks, Ubiquitous, and Trustworthy Computing, 2006., vol.1, pp.8 pp., 5-7 June 2006 doi:10.1109/SUTC.2006.1636181

[15] Johnston, M.G., "Ground Object Geo-Location using UAV Video Camera," 25th Digital Avionics Systems Conference, 2006 IEEE/AIAA, pp.1-7, 15-19 Oct. 2006 doi: 10.1109/DASC.2006.313770

[16] Rally Maps. Available at: http://www.rally-maps.com/map (last accessed May 3, 2011)

[17] Vidmap. Available at: http://www.vidmap.de/ (last accessed May 3, 2011)

[18] Qik. Available at: http://qik.com/ (last accessed May 3, 2011)

[19] Ipoki. Avalilable at: http://www.ipoki.com/ (last accessed May 3, 2011)

[20] Gat Y., Kozintsev I., Nestares O., "Fusing image data with location and orientation sensor data streams for consumer video applications," Computer Vision and Pattern Recognition Workshops (CVPRW), 2010 IEEE Computer Society Conference on , vol., no., pp.1-8, 13-18 June 2010 doi: 10.1109/CVPRW.2010.5543781

[21] Rivest, R., Shamir, A., and Adleman, L. "A method for obtaining digital signatures and public-key cryptosystems”, Communications of the ACM, 21, 2 (Feb. 1978), 120-126. http://doi.acm.org/10.1145/359340.359342

\section{Copyright Disclaimer}

Copyright reserved by the author(s).

This article is an open-access article distributed under the terms and conditions of the Creative Commons Attribution license (http://creativecommons.org/licenses/by/3.0/). 